Rikei Kozakai, Akira Kushida, Paul Franck Adeyissimi Adjou Moumouni, Sadatsugu Okuma, Kazuya Takahashi, Kuniko Hoshi, Yuri Sato, Mizue Takahashi, Nodoka Chida, Mei Takahashi, Shukuko Iwabuchi, Yoshihiko Izumi, Kana Fukami, Shuji Nakashiro, Hisashi Nojima and Shinichiro Takahashi*

\title{
Assessment of COVID-19 mRNA vaccination titer and side effects in healthy volunteers
}

https://doi.org/10.1515/labmed-2021-0156

Received October 22, 2021; accepted December 14, 2021; published online January 3, 2022

\section{Abstract}

Objectives: An effective vaccine against SARS-CoV-2 is essential to mitigate the COVID-19 pandemic. In these several months, a number of groups have started to report humoral responses and side effects after BNT162b2 vaccinations. Although these reports demonstrate the safety and efficacy, further studies are warranted to verify these findings. Here we examined the levels of SARS-CoV-2 antibodies in Japanese healthy volunteers who underwent BNT162b2 vaccine, to assess the humoral responses and side effects.

Methods: Forty-one healthy volunteers' samples were used for the measurement of SARS-CoV-2 antibodies with chemiluminescent assays against the Receptor Binding Domain (RBD) of the virus. We also measured the side effects of the vaccination.

Results: Although the levels of IgM varied, all participants were seronegative for IgM and IgG before vaccination, and both IgM and IgG were significantly increased after the vaccinations. We further analyzed the humoral responses in relation to age, and found that the IgG levels for 14 days and 35 days, and IgM levels for 14 days after vaccination showed clear declining trends with age. Commonly reported side effects in the participants were sore arm/pain

\footnotetext{
*Corresponding author: Shinichiro Takahashi, Division of Laboratory Medicine, Faculty of Medicine, Tohoku Medical and Pharmaceutical University, Sendai, Japan, Phone: +81-22-290-8889,

E-mail: shintakahashi@tohoku-mpu.ac.jp

Rikei Kozakai, Kuniko Hoshi, Yuri Sato, Mizue Takahashi, Nodoka Chida, Mei Takahashi, Shukuko Iwabuchi and Yoshihiko Izumi, Department of Clinical Laboratory, Tohoku Medical and Pharmaceutical University Hospital, Sendai, Japan

Akira Kushida, Kana Fukami and Shuji Nakashiro, Product Planning Department, Fujirebio Inc., Tokyo, Japan

Paul Franck Adeyissimi Adjou Moumouni, Sadatsugu Okuma and Hisashi Nojima, R\&D Strategy Planning Section, Fujirebio Inc., Tokyo, Japan

Kazuya Takahashi, Product Development Department Product Designing 4th Section, Fujirebio Inc., Tokyo, Japan
}

(90.0\%) after the first dose, and generalized weakness/ fatigue (70.0\%), fever (57.5\%), and sore arm/pain (90.0\%) after the second dose.

Conclusions: BNT162b2 vaccination generates sufficient production of IgG especially after the second dose, though the response decreases age-dependently. The high frequencies of generalized weakness/fatigue, fever, and sore arm/pain were not negligible, especially after the second dose. This may be associated with the age characteristics of the population.

Keywords: BNT162b2 vaccine; humoral response; SARS-CoV2; side effects; vaccination.

\section{Introduction}

As of the end of October 2021, severe acute respiratory syndrome coronavirus-2 (SARS-CoV-2) has infected over 242 million individuals worldwide, resulting in more than 4.92 million deaths. Many vaccines are now being employed to prevent the novel coronavirus disease 2019 (COVID-19) caused by SARS-CoV-2 infection, including RNA vaccines [1]. BNT162b2 (Comirnaty; BioNTech/ Pfizer) is a lipid nanoparticle-formulated, nucleosidemodified mRNA vaccine for the prevention of COVID-19 [2]. The BNT162b2 vaccine was investigated in the present study because it was the only vaccine approved by the Ministry of Health, Labour and Welfare, Japan, when the study protocol was planned. In clinical studies of BNT162b2, the geometric mean titer of neutralizing antibodies associated with 50\% neutralization increased after the first dose, and the vaccine provided high levels of protection against infection and severe disease after the second dose [3]. In a randomized, placebo-controlled clinical trial involving approximately 44,000 participants, immunization conferred $95 \%$ protection against COVID-19 [4]. Related mRNA vaccines also appear to be safe and effective [5, 6]. Recently, humoral responses in three volunteers after BNT162b2 vaccination were reported by Danese et al. [7]. They also reported that prominent humoral immune responses, especially 
involving anti-SARS-CoV-2 IgG and IgA, were intensified by a second vaccination dose [7]. In these several months, a number of groups have started to report humoral responses and side effects after BNT162b2 vaccinations [8-10]. Although these reports demonstrate the safety and efficacy, further studies are warranted to verify these findings. The present study enrolled 41 healthy Japanese healthcare worker volunteers to determine the antibody production after BNT162b2 vaccination based on antiSARS-CoV-2 titers. These titers were evaluated using Lumipulse platform, which is highly sensitive and fully automated assay system based on a chemiluminescent enzyme immunoassay (CLEIA) to specifically detect IgM and $\operatorname{IgG}$, especially those recognizing the receptorbinding domain (RBD) of the SARS-CoV-2 spike protein, which is involved in ACE2-mediated viral entry.

\section{Materials and methods}

\section{Participants}

The vaccination campaign for administration of $30 \mu \mathrm{g}$ of BNT162b2 vaccine (Comirnaty; BioNTech/Pfizer, New York, NY, USA) began in Tohoku Medical and Pharmaceutical University Hospital on 15 March 2021. Forty-one healthy volunteers were enrolled in the present study. They comprised 31 female and 10 male healthcare workers who received a first BNT162b2 vaccine dose from March to April 2021, followed by a second identical dose 21 days later. All the blood samples were collected before 1-3 days before vaccination, two weeks after first vaccination, and two weeks after second vaccination (Figure 1A). Then the samples were centrifuged at $1,690 \times \mathrm{g}$ for $10 \mathrm{~min}$ at room temperature, and the serum was separated for storage in two 1-mL aliquots at $-80^{\circ} \mathrm{C}$. The study design was approved by the Ethics Committee at the Tohoku Medical and Pharmaceutical University Hospital in the 2020 fiscal year (2020-2-256).

A

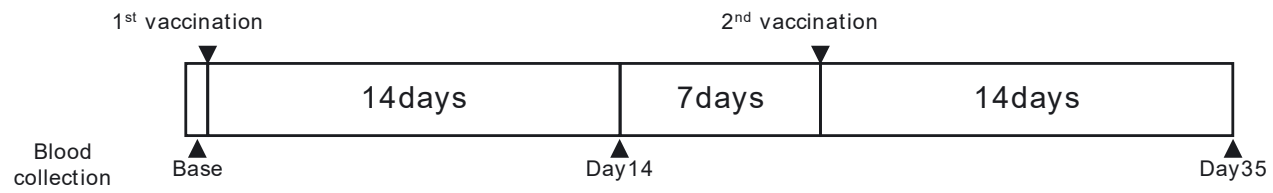

B

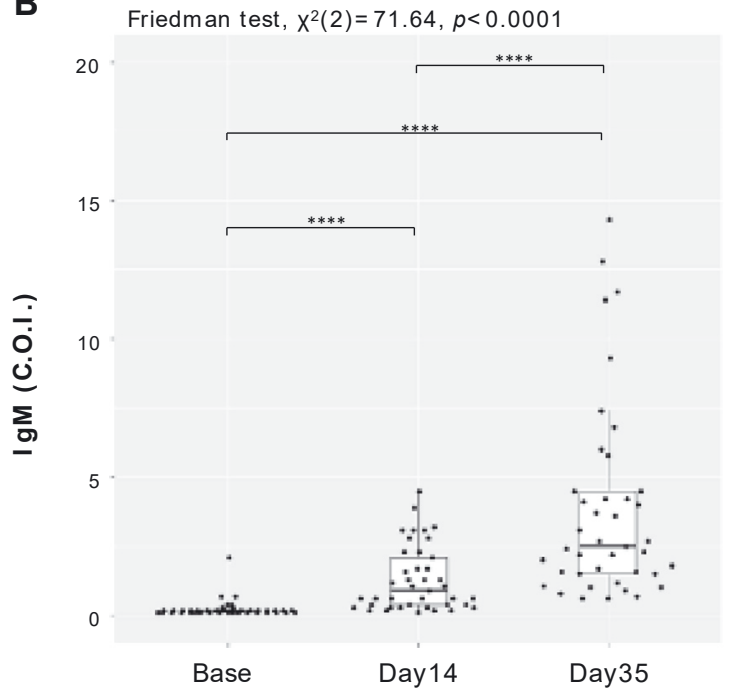

Days after first vaccination

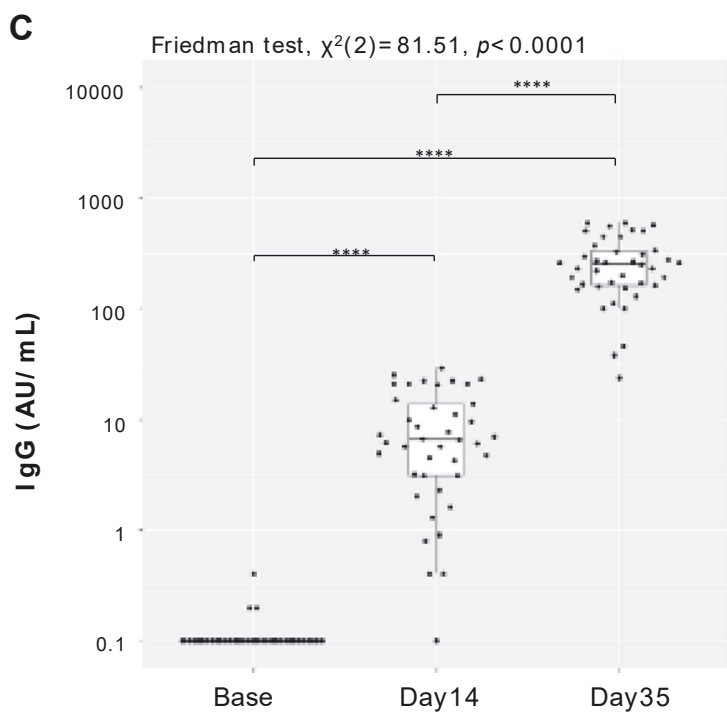

Days after first vaccination

Figure 1: Vaccination procedures, sampling and anti-SARS-CoV-2 antibody levels following vaccination.

(A) Schematic presentation of vaccination and sampling procedures. (B, C) Box plots of anti-SARS-CoV-2 antibody levels following vaccination with the BNT162b2 vaccine. (B) Anti-SARS-CoV-2 IgM at baseline, and at 14 and 35 days after the first vaccination in all participants. (C) AntiSARS-CoV-2 IgG at baseline, and at 14 and 35 days after the first vaccination in all participants. Box plots display the median values with the interquartile range (lower and upper hinge) and \pm 1.5 -fold the interquartile range from the first and third quartile (lower and upper whiskers). Data were analyzed using the Friedman test followed by pairwise Wilcoxon signed rank tests with Bonferroni correction. ${ }^{\star \star \star \star} p<0.0001$. 


\section{Safety assessments}

Safety assessments were monitored by solicited local and systemic adverse events, collected by a questionnaire. Items for symptoms and events were utilized and modified from the study by Kadali et al. [11]. The questionnaire was sent by e-mail to the participants enrolled in the study, and responses were collected until the middle of May 2021. Responses were obtained from 40 of the 41 healthy healthcare volunteers (Table 1) who received two doses of the BNT162b2 vaccine. For analysis of the questionnaire data, the responses were displayed as both percentage and number.

\section{Antibody assays}

All serum aliquots were thawed at the same time for analysis at the end of the study, and examined using SARS-CoV-2 S-IgG and SARS-CoV-2 S-IgM reagents (Fujirebio, Tokyo, Japan) for the in vitro quantitative determination of antibodies to the SARS-CoV-2 spike (S) protein receptor binding domain (RBD), according to the manufacturer's instructions. The levels of IgG or IgM were expressed in arbitrary units $(\mathrm{AU}) / \mathrm{mL}$ or evaluated in accordance with a cut-off index (C.O.I.), respectively, which were calculated by quantification of the luminescence in the samples. The assay results are interpreted as reactive $(>1.0 \mathrm{AU} / \mathrm{mL}$ or $>1.0$ C.O.I; positive) and non-reactive $(<1.0 \mathrm{AU} / \mathrm{mL}$ or $<1.0$ C.O.I.; negative) as shown in the manufacturer's instructions. Dilutional linearity experiments were performed with the samples; high concentrated serums were diluted using the anti-SARS-CoV2 antibody negative serums.

\section{Statistical analysis}

Humoral immune response data were statistically analyzed using Prism software (version 7.0; GraphPad Software Inc., La Jolla, CA, USA) or

Table 1: Demographic data of the study participants.

\begin{tabular}{lrr}
\hline & Percentage, $\%$ & Number, $\mathbf{n}$ \\
\hline Age group, years & & \\
$21-30$ & 31.7 & 13 \\
$31-40$ & 29.3 & 12 \\
$41-50$ & 12.2 & 5 \\
$51-60$ & 17.0 & 7 \\
$61-70$ & 9.8 & 4 \\
Gender & & \\
Female & 75.6 & 31 \\
$\quad$ Male & 24.4 & 10 \\
Previous infection with SARA-CoV-2 & & \\
$\quad$ Infected & 0.0 & 0 \\
$\quad$ Not infected & 100.0 & 41 \\
Type of COVID-19 vaccine & & \\
$\quad$ Pfizer BNT162b2 vaccine & 100.0 & 41 \\
Inoculated vaccine dose & & \\
1st Dose & 0.0 & 0 \\
1st and 2nd Dose & 100.0 & 41 \\
\hline
\end{tabular}

$\mathrm{R}$ version 4.0.5 (R Foundation for Statistical Computing). The level of significance was set at $\mathrm{p} \leq 0.05$. Correlations between Age and the levels of IgM or IgG were examined with Spearman's correlation test. Group differences in the antibodies' levels were first tested with Friedman or Kruskal-Wallis test and, when statistically significant, further investigated with the Wilcoxon signed-rank test or the Wilcoxon rank-sum test. For group comparisons, $\mathrm{p}$-values were corrected using the Bonferroni or the Steel-Dwass method.

\section{Results}

Specificity of the antibody assays were confirmed using SARS-CoV-2 negative sera which were collected before 2019 , resulting in $100 \%(1,011 / 1,011)$ for SARS-CoV-2 S-IgG reagents and $99.8 \%(1,056 / 1,058)$ for SARS-CoV-2 S-IgM reagents (Supplementary Figure S1A-B). Dilutional linearity was performed with samples which were within the working range and showed reasonable results (Supplementary Figure S1 C-D). The reproducibility tests of the kits resulted in reliable outcomes (Supplementary Table S1). The cumulative results for antibody production are shown in Figure 1, panels B-C. Although there were no obvious symptoms or history of COVID-19 infection, one of the 41 participants had a marginally increased level of SARS-CoV-2 IgM (2.1 C.O.I.) before vaccination (Figure 1B), which we considered to be within the variation of seronegative, and all subjects had baseline levels of SARS-CoV-2 IgG (Figure 1C).

At 14 days after the first vaccination, anti-SARS-CoV-2 IgM was modestly but significantly increased (average, 6.5-fold) (Figure 1B), while anti-SARS-CoV-2 IgG was more markedly increased (average, 93-fold) (Figure 1C). At 35 days after the first vaccination, anti-SARS-CoV-2 IgM was significantly increased (average, 19.5-fold) (Figure 1B), while anti-SARS-CoV-2 IgG was prominently enhanced (average, 2719-fold) (Figure 1C). We further analyzed the humoral responses in relation to age, and found that the IgG levels for 14 days and 35 days, and IgM levels for 14 days after vaccination showed clear declining trends with age, though an age difference was not seen in the IgM levels at 35 days after the vaccination (Figure 2 and Supplementary Figure S2). We further analyzed the differences between males and females, but no significant difference in both IgG and IgM titers were observed (data not shown).

Next, we assessed the side effects after vaccination (Figure 3 and Supplementary Table S2). Throughout the study, almost all participants (90\% after the first dose; 


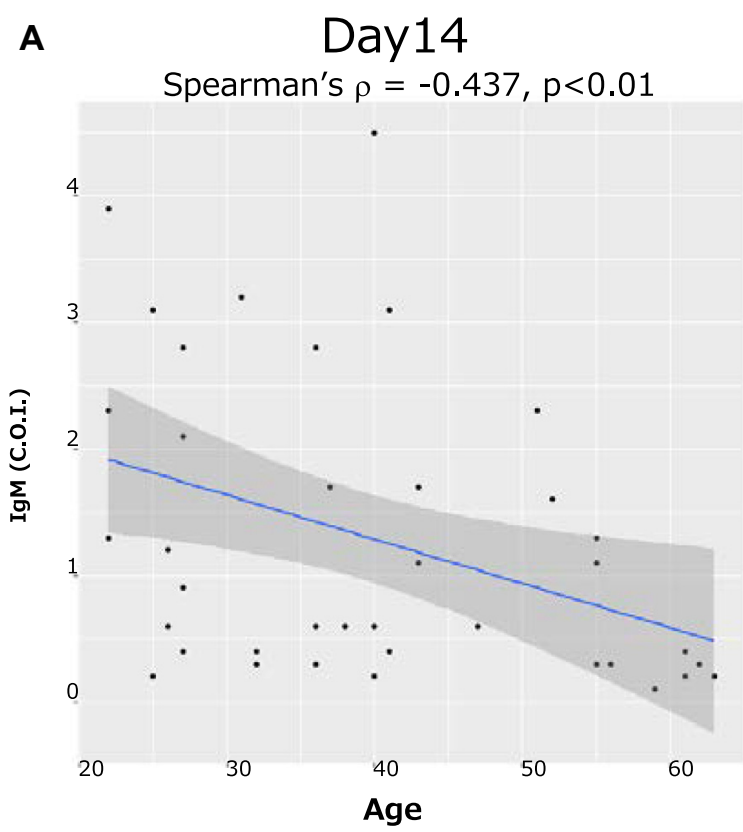

B
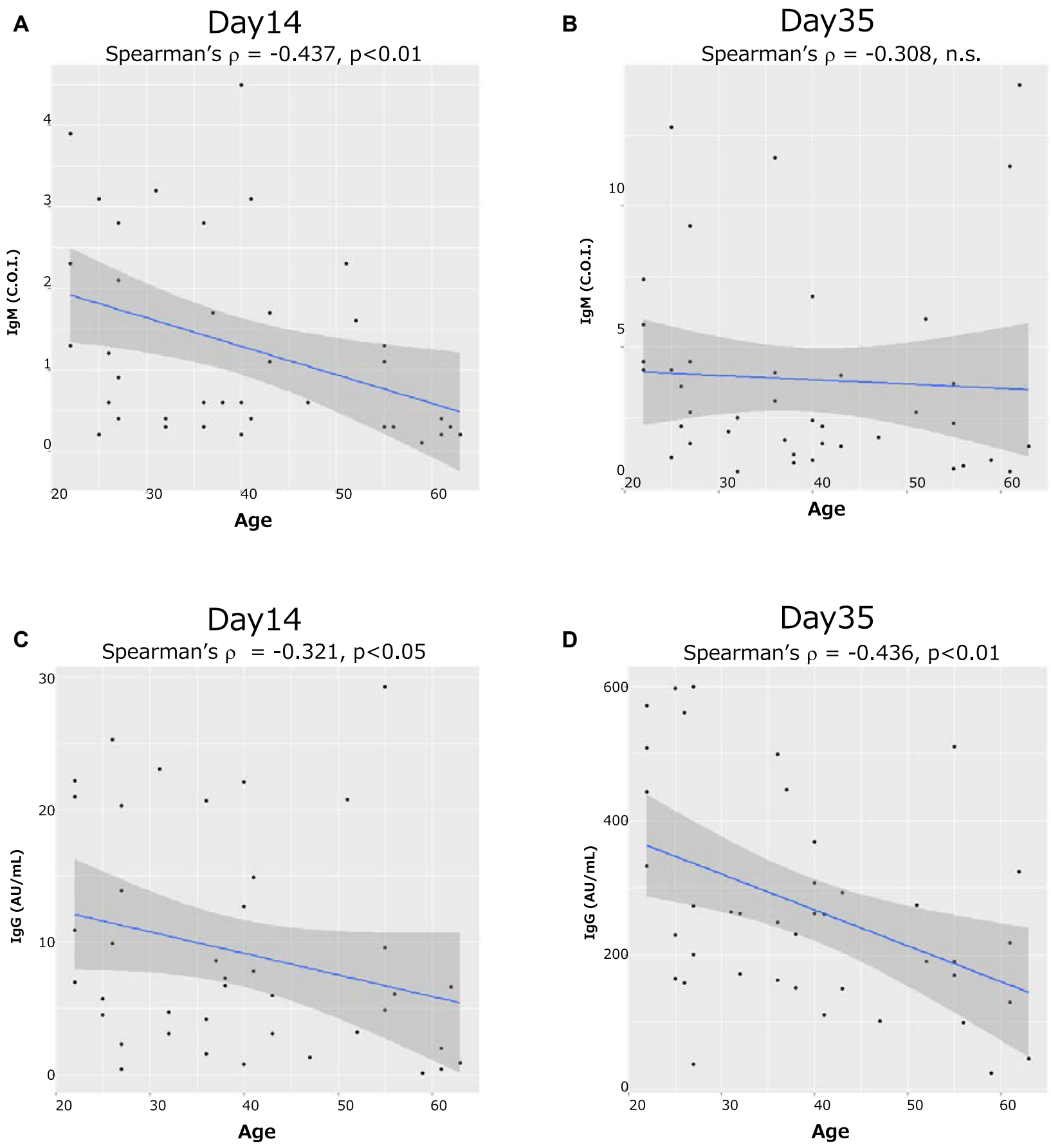

Figure 2: Correlation of age and post-vaccination at day 14 (A, C) and day 35 (B, D) of anti-Spike IgM (A, B) or IgG (C, D) levels with superimposed the linear regression lines (with $95 \% \mathrm{Cl}$ ).

The statistical methods of Spearman's rank correlation coefficient were used. p-Value is indicated. Linear regression lines, blue.

97.5\% after the second dose) had symptoms (Figure 4A). The total number of side effects was significantly increased after the second vaccination, compared with the first vaccination (Figure 4B). We further analyzed whether there were any differences in the numbers of side effects among the age groups, but no significant differences were found (Figure 4C and D, Supplementary Figure S3). 

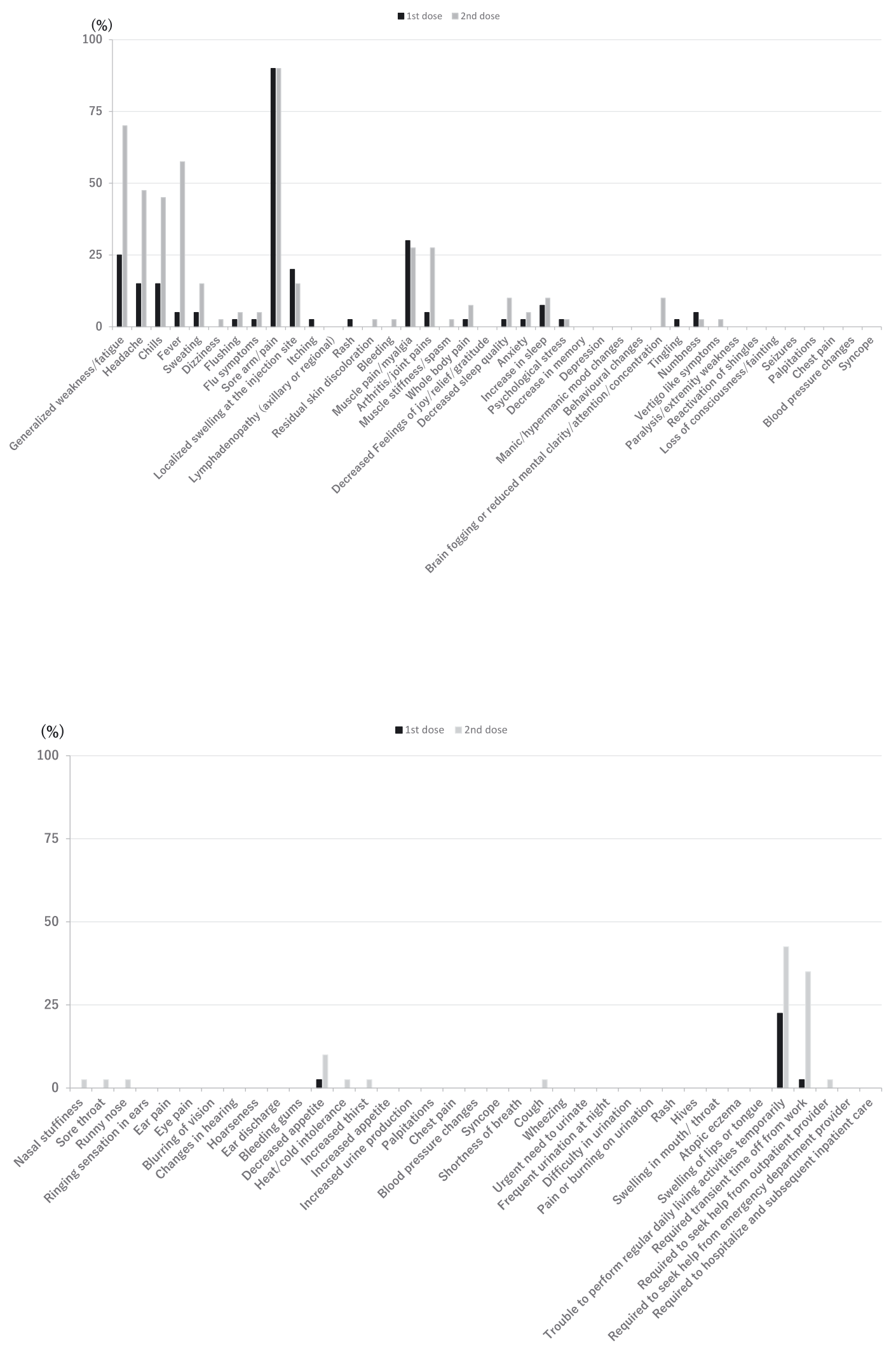

Figure 3: Summary of the side effects of the BNT162b2 vaccine after the first (black bars) and second (gray bars) doses of the vaccine. The data are shown as frequency (\%). 

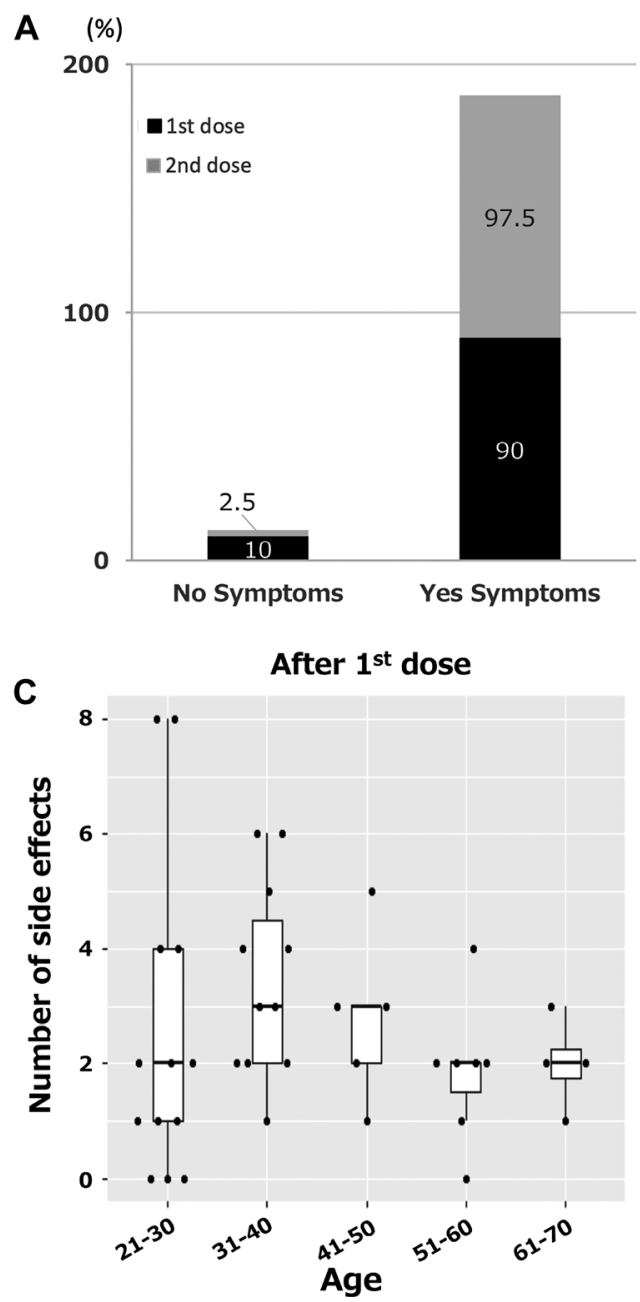

B
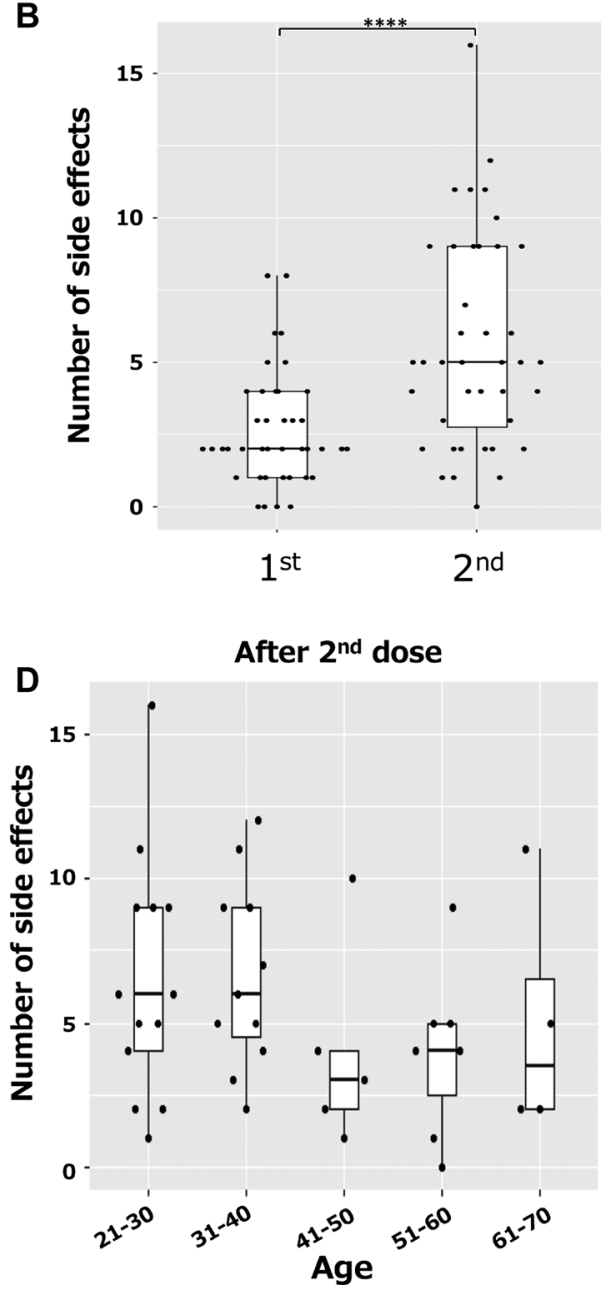

Figure 4: Summary of the side effects of the BNT162b2 vaccine.

(A) Percentages for the presence (symptoms) or absence (no symptoms) of the side effects of the BNT162b2 vaccine reported by the participants after the first and second doses of the vaccine. (B) Box plots of total numbers of side effects in individual participants after the first and second doses. (C, D) Box plots of the total numbers of side effects in individual participants, categorized into five different age groups, at first dose (C), and second dose (D). Box plots display the median values with the interquartile range (lower and upper hinge) and \pm 1.5 -fold the interquartile range from the first and third quartile (lower and upper whiskers). Data were analyzed using the Wilcoxon rank-sum test. $\star \star \star \star p<0.0001$.

\section{Discussion}

Side effects of the BNT162b2 vaccine have been reported by several groups [10-12]. In a study on 803 healthcare workers who received the BNT162b2 vaccine [11], $92.9 \%$ of the workers received the first and second doses, and were evaluated for side effects. The most common side effects of the vaccine were sore arm/pain (88.04\%), generalized weakness/fatigue (58.89\%), muscle pain/myalgia (45.70\%), headache (45.48\%), chills (36.60\%), and fever (21.99\%). In the present study conducted on Japanese subjects, the types of side effects did not differ significantly from those in the previous reports, but there were some differences in their frequency. BNT162b2 was supposed to show a lower incidence and severity of systemic reactions, particularly in older adults $[6,10]$. In the present study, however, we observed a clearly higher rate of fever (57.5\%) after the second dose. In addition, there were no significant differences between side effects and age group (Figure $4 \mathrm{C}$ and $\mathrm{D}$ ). These differences may have arisen from the sample size or generational differences; the relatively younger age of the population of volunteers enrolled in the present study (Table 1) compared with the other studies [10-12].

Published information on humoral responses to BNT162b2 remains limited. In the present study, we observed insufficient induction of both IgM and IgG titers 
after the first vaccine dose (Figure 1). These results are consistent with a study showing that a single BNT162b2 vaccine dose in naïve subjects induced low levels of neutralizing antibodies $[13,14]$. In the present study, the participants obtained high levels of anti-SARS-CoV-2 RBD IgG at 2 weeks after the second vaccination, consisting with the findings in previous reports $[6,15,16]$. These findings suggest that vaccination with both the first and second doses is necessary to achieve sufficient effects.

The present observation of low anti-SARS-CoV-2 RBD IgM titers after vaccination is consistent with the results from other groups $[17,18]$. These findings suggest that the IgM antibody class may have limited importance for neutralization of SARS-CoV-2. We consider that IgG, but not IgM, is suitable for evaluating the response to vaccination, because of the high titers.

Although only a few data on the IgM antibody response after BNT162b2 vaccination are available, Beharier et al. [19] recently published an analysis of maternal-to-neonatal transfer of antibodies elicited by the BNT162b2 vaccine. In their analysis of 213 participants (vaccinated: $n=86$; infected: $n=65$; unvaccinated non-infected controls: $n=62$ ), the levels of anti-RBD IgM in unvaccinated non-infected controls varied from approximately 1 to $4 \log 10$ mean fluorescence intensity (MFI). However, most converged at $<3.5$, which we consider to be approximately equal to our quantitative unit C.O.I. We observed a substantially high SARS-CoV-2 IgM level in one participant (Figure 1B), and several cases above $4 \log 10$ MFI were similarly observed in the previous study [19]. In our separate experiment, 1,056 of 1,058 negative samples were below 1 C.O.I., indicating a specificity of $99.8 \%$ for this reagent (Supplementary Figure S1). IgM has been reported to have a non-specific response, as represented by (but not limited to) rheumatoid factor [20], therefore, the IgM assay, as in this reagent, may be more susceptible to interferents than the $\operatorname{IgG}$ assay. In fact, inhibition assay using anti-human IgM antibody resulted in a decrease in the measured values of this supposedly pseudo-high sample, suggesting that a non-specific reaction by IgM was the probable reason (data not shown).

We demonstrated that there was a significant inverse correlation between anti-RBD IgG and age (Figure 2) and the similar trend has reported $[6,8,14]$. Several factors have been demonstrated to affect anti-SARS-CoV-2 antibody titers. A study in the United States reported that mRNA vaccines generally showed lower virus-neutralizing responses in participants aged 65-85 years compared with those aged 18-55 years [6]. In Germany, Müller et al. [8] demonstrated that the SARS-CoV-2 spike-specific IgG titer was significantly lower in after both the first and second vaccinations in those aged $\geq 80$ years. In the present study on Japanese subjects, the results were similar to those previously reported in the United States and Germany. In contrast, Watanabe et al. [9] recently investigated an Italian study population and reported that higher waist circumference, smoking, hypertension, and longer elapsed time after second vaccination were associated with lower antibody titers. In their report [9], BMI, age, and sex were not related to antibody production. We consider that these discrepancies arise from differences in the population characteristics (age, weight, waist circumference tendency) of the healthcare workers in the different countries involved in the studies. It is also worth noting that antibody titers tend to decline rapidly following vaccination with kinetics that have not been completely clarified and may potentially be different in some populations [16].

In this study, we report for the first time the changes in antibody titers over time in Japanese patients before and after vaccination determined using the newly developed Lumipulse detection system. Although a variety of reagents have been used to measure anti-SARS-CoV-2 antibodies, the fact that the Lumipulse reagents were able to confirm the acquisition of antibodies after vaccination suggests that they can measure anti-SARS-CoV-2 antibodies appropriately. The Lumipulse reagents may be useful in such studies. Timely monitoring by employing these antibodies could be one of the efficient tools to conquer the ongoing COVID-19 pandemic.

Acknowledgments: The authors thank to generous help for all the members in the Department of Clinical Laboratory in Tohoku Medical and Pharmaceutical Hospital. The authors also appreciate Mr. T Moromi, Mr. M Matsumoto in Fujirebio, for helpful discussion and support. Finally, the authors thank Alison Sherwin, PhD, from Edanz (https://jp. edanz.com/ac) for editing a draft of this manuscript.

Research funding: This work was supported in part by the Shino-Test Corporation Research Fund.

Author contributions: All authors have accepted responsibility for the entire content of this manuscript and approved its submission. R.K. and S.T. designed the study. R.K., K.H., Y.S., M.T., N.C., M.T., S.I. and Y.I. provided samples and clinical data. K.T., A.K., P.A., S.O., K.F., S.N., H.N. provided the data of antibody assays' performance. R.K., H.N. and S.T. performed statistical analysis. R.K., H.N. and S.T. prepared the manuscript. All authors participated in discussions and interpretation of the data and results.

Competing interests: A.K., P.A., S.O., K.T., K.F., S.N., H.N.: full-time employee of FUJIREBIO Co., R.K., K.H., Y.S., M.T., N.C., M.T., S.I., Y.I.: no potential competing interest, S.T.: granted from Shino-Test Corp. research fund. Outside the 
submitted work, S.T. is granted from Grant-in-Aid for Scientific Research (21K07346) from the Ministry of Education, Science and Culture, Japan, Daiichi-Sankyo Research Support, Kyowa-Kirin Research Support.

Informed consent: Informed consent was obtained from all individuals included in this study.

Ethical approval: Research involving human subjects complied with all relevant national regulations, institutional policies and is in accordance with the tenets of the Helsinki Declaration (as revised in 2013), and has been approved by the Ethics Committee at the Tohoku Medical and Pharmaceutical University Hospital in the 2020 fiscal year (2020-2-256).

\section{References}

1. Topol EJ. Messenger RNA vaccines against SARS-CoV-2. Cell 2021; 184:1401.

2. Lamb YN. BNT162b2 mRNA COVID-19 vaccine: first approval. Drugs 2021;81:495-501.

3. Collier DA, De Marco A, Ferreira I, Meng B, Datir RP, Walls AC, et al. Sensitivity of SARS-CoV-2 B.1.1.7 to mRNA vaccine-elicited antibodies. Nature 2021;593:136-41.

4. Polack FP, Thomas SJ, Kitchin N, Absalon J, Gurtman A, Lockhart S, et al. Safety and efficacy of the BNT162b2 mRNA COVID-19 vaccine. N Engl J Med 2020;383:2603-15.

5. Baden LR, El Sahly HM, Essink B, Kotloff K, Frey S, Novak R, et al. Efficacy and safety of the mRNA-1273 SARS-CoV-2 vaccine. N Engl J Med 2021;384:403-16.

6. Walsh EE, Frenck RW Jr., Falsey AR, Kitchin N, Absalon J, Gurtman A, et al. Safety and immunogenicity of two RNA-based COVID-19 vaccine Candidates. N Engl J Med 2020;383:2439-50.

7. Danese E, Montagnana M, Salvagno GL, Peserico D, Pighi L, De Nitto $S$, et al. Comprehensive assessment of humoral response after Pfizer BNT162b2 mRNA COVID-19 vaccination: a three-case series. Clin Chem Lab Med 2021;59:1585-91.

8. Muller L, Andree M, Moskorz W, Drexler I, Walotka L, Grothmann R, et al. Age-dependent immune response to the Biontech/Pfizer BNT162b2 COVID-19 vaccination. Clin Infect Dis 2021 Apr 27. https://doi.org/10.1093/cid/ciab381 [Epub ahead of print].

9. Watanabe M, Balena A, Tuccinardi D, Tozzi R, Risi R, Masi D, et al. Central obesity, smoking habit, and hypertension are associated with lower antibody titres in response to COVID-19 mRNA vaccine. Diabetes Metab Res Rev 2021:e3465. https://doi.org/10.1002/ dmrr.3465.

10. Izumo T, Kuse N, Awano N, Tone M, Sakamoto K, Takada K, et al. Side effects and antibody titer transition of the BNT162b2 messenger ribonucleic acid coronavirus disease 2019 vaccine in Japan. Respir Investig 2021;59:635-42.

11. Kadali RAK, Janagama R, Peruru S, Malayala SV. Side effects of BNT162b2 mRNA COVID-19 vaccine: a randomized, crosssectional study with detailed self-reported symptoms from healthcare workers. Int J Infect Dis 2021;106:376-81.

12. El-Shitany NA, Harakeh S, Badr-Eldin SM, Bagher AM, Eid B, Almukadi $\mathrm{H}$, et al. Minor to moderate side effects of Pfizer-BioNTech COVID-19 vaccine among Saudi residents: a retrospective crosssectional study. Int J Gen Med 2021;14:1389-401.

13. Gobbi F, Buonfrate D, Moro L, Rodari P, Piubelli C, Caldrer S, et al. Antibody response to the BNT162b2 mRNA COVID-19 vaccine in subjects with prior SARS-CoV-2 infection. Viruses 2021;13:422.

14. Padoan A, Dall'Olmo L, Rocca FD, Barbaro F, Cosma C, Basso D, et al. Antibody response to first and second dose of BNT162b2 in a cohort of characterized healthcare workers. Clin Chim Acta 2021; 519:60-3.

15. Jackson LA, Anderson EJ, Rouphael NG, Roberts PC, Makhene M, Coler RN, et al. An mRNA vaccine against SARS-CoV-2 preliminary report. N Engl J Med 2020;383:1920-31.

16. Sahin U, Muik A, Derhovanessian E, Vogler I, Kranz LM, Vormehr $M$, et al. COVID-19 vaccine BNT162b1 elicits human antibody and TH1 T cell responses. Nature 2020;586:594-9.

17. Wang Z, Schmidt F, Weisblum Y, Muecksch F, Barnes CO, Finkin S, et al. mRNA vaccine-elicited antibodies to SARS-CoV- 2 and circulating variants. Nature 2021;592:616-22.

18. Mazzoni A, Di Lauria N, Maggi L, Salvati L, Vanni A, Capone M, et al. First-dose mRNA vaccination is sufficient to reactivate immunological memory to SARS-CoV-2 in subjects who have recovered from COVID-19. J Clin Invest 2021;131:e149150.

19. Beharier O, Plitman Mayo R, Raz T, Nahum Sacks K, Schreiber L, Suissa-Cohen $\mathrm{Y}$, et al. Efficient maternal to neonatal transfer of antibodies against SARS-CoV-2 and BNT162b2 mRNA COVID-19 vaccine. J Clin Invest 2021;131:e154834.

20. Bjerner J. Human anti-immunoglobulin antibodies interfering in immunometric assays. Scand J Clin Lab Invest 2005;65:349-64.

Supplementary Material: The online version of this article offers supplementary material (https://doi.org/10.1515/labmed-2021-0156). 